\title{
Reconstrução de trânsito intestinal: fatores que influenciam a realização
}

\author{
Janderson Cleiton Aguiar ${ }^{1}$, \\ Adriana Pelegrini dos Santos Pereira², \\ Maria Helena Pinto ${ }^{3}$
}

\section{RESUMO}

Objetivou-se identificar os fatores que influenciam na reconstrução do trânsito intestinal em pessoas com estoma provisório. Trata-se de um estudo descritivo-analítico, quantitativo, participaram 117 pessoas com estoma provisório, que foram entrevistados, abordando aspectos sociodemográficos, clínicos e motivo da não reconstrução do estoma até o momento da coleta dos dados, foi realizada análise descritiva das variáveis, com aplicação de testes para verificar a existência de relação entre os motivos de demora para reconstrução com as demais variáveis. A maioria era do sexo masculino, a neoplasia predominou como patologia que determinou a construção do estoma, as cirurgias foram de urgência, sendo a colectomia a mais frequente. A idade influencia tanto na causa do estoma como na não reconstrução, assim como a presença de comorbidades e a persistência da causa pré-cirúrgica, o que leva à reflexão sobre a importância de ações de promoção de saúde e prevenção de doenças intestinais.

Descritores: Estomia; Estomas Cirúrgicos; Cuidados de Enfermagem; Perfil de Saúde.

\footnotetext{
${ }^{1}$ Enfermeiro, Mestre em Enfermagem. Professor da União das Faculdades dos Grandes Lagos. São José do Rio Preto, SP, Brasil. E-mail: janderpoty@yahoo.com.br.

${ }^{2}$ Enfermeira, Doutora em Ciências da Saúde. Professora Assistente do Departamento de Enfermagem Geral da Faculdade de Medicina de São José do Rio Preto. São José do Rio Preto, SP, Brasil. E-mail: adrianapelegrini@famerp.br.

${ }^{3}$ Enfermeira, Doutora em Enfermagem Fundamental. Professora Adjunta do Departamento de Enfermagem Geral da Faculdade de Medicina de São José do Rio Preto. São José do Rio Preto, SP, Brasil. E-mail: mariahelena@famerp.br.
}

Artigo recebido: 28/06/2017.

Artigo aprovado: 27/04/2018.

Artigo publicado: 13/12/2018.

Como citar esse artigo:

Aguiar JC, Pereira APS, Pinto MH. Reconstrução de trânsito intestinal: fatores que influenciam a realização. Rev. Eletr. Enf. [Internet]. 2018 [acesso em: ];20:v20a32. Disponível em: https://doi.org/10.5216/ree.v20.47606. 


\section{INTRODUÇÃO}

A construção de estomas intestinais é comum em cirurgias do trato gastrointestinal e possuem como principal finalidade a eliminação de fezes e gazes ${ }^{(1)}$. Sua nomenclatura varia de acordo com o órgão ou porção do mesmo que foi exteriorizado, sendo denominado de ileostomia quando há exteriorização do segmento ileal e colostomia quando se refere ao segmento cólico(1).

O estoma intestinal pode ser confeccionado decorrente de diversas doenças e suas complicações, como fístulas e abdômen agudo, este podendo ser do tipo obstrutivo, causado em virtude de neoplasias, volvo e megacolon, entre outras, ou perfurativo, decorrente principalmente de neoplasias, doenças diverticulares, doenças inflamatórias intestinais e traumas. Não obstante, o cirurgião também pode optar pela técnica de construção de um estoma anterior a uma anastomose para sua proteção(1).

De acordo com localização, órgão e causa de confecção do estoma, o cirurgião pode considerar e optar pela técnica cirúrgica mais benéfica de acordo com cada caso ${ }^{(1)}$. A técnica definitiva é quando o paciente não apresenta possibilidade de reconstrução do trânsito intestinal após a realização de um estoma, já quando há possibilidade de reconstrução denomina-se de provisórios. No entanto, mesmo diante da possibilidade da reconstrução do trânsito intestinal a mesma nem sempre é realizada, decorrente de fatores individuais intrínsecos e extrínsecos a este procedimento, como a causa do estoma, técnica cirúrgica empregada e presença de comorbidades, refletindo na reconstrução, impactando nas taxas de morbimortalidade ${ }^{(1-4)}$. Considerando os índices de morbimortalidade na reconstrução de transito intestinal é prudente avaliar a necessidade de confecção de um estoma, restringindo a sua confecção quando possível, especialmente aos estomas em boca terminal do tipo Hartmann, visto suas taxas de complicações na reconstrução(5).

Entretanto, os pacientes submetidos a estomas intestinais, necessitam de bolsa coletora, as quais demandam cuidados específicos com a pele, higienização e troca da bolsa. A presença da mesma pode gerar alteração da imagem corporal e modificações das atividades rotineiras, levando a pessoa a experimentar sentimentos negativos sobre si, redução da autoestima, insatisfação com a vida, incerteza quanto ao futuro e medo de rejeição(6-7). Desta maneira, tais fatores também devem ser considerados na avaliação para a realização deste procedimento.

É importante ressaltar que a avaliação para a reconstrução do trânsito intestinal perpassa pelas recomendações contidas nas diretrizes para atenção à saúde das pessoas com estoma no âmbito do Sistema Único de Saúde (SUS), as quais estabelecem que as atribuições dos serviços de atenção aos estomizados relacionam-se à capacitação das equipes assistenciais, à atualização constante do cadastro das pessoas com estoma e ao acompanhamento dos mesmos desde a fase pré-operatória até a reconstrução do trânsito intestinal ${ }^{(8)}$. Neste cenário, tais elementos possibilitam monitorizar os fatores que podem influenciar no procedimento de reconstrução de trânsito, proporcionando planejamento assistencial adequado, reconstrução intestinal em menor tempo de acordo com cada caso e a reabilitação dos mesmos de forma mais efetiva.

Em virtude do exposto, o objetivo do estudo foi identificar os fatores que influenciam na reconstrução do trânsito intestinal em pessoas com estoma provisório.

\section{MÉTODO}

Estudo descritivo analítico de caráter quantitativo realizado com pessoas com estoma intestinal provisório atendidos em um Serviço de Atenção ao Estomizado localizado no interior do Estado de São Paulo. Este serviço atende uma região de 90 municípios, composta pelo município sede do serviço, uma microrregião formada por 30 municípios 
próximos e uma macrorregião composta por 59 municípios situados geograficamente distantes da sede do serviço. Possui equipe composta por médico coloproctologista, enfermeiro estomaterapeuta, nutricionista, psicólogo e assistente social que prestam assistência especializada e de natureza interdisciplinar às pessoas com estoma, objetivando a reabilitação, incluindo a orientação para o autocuidado, prevenção e tratamento de complicações nas estomias, capacitação e fornecimento de equipamentos coletores e adjuvantes de proteção e segurança ${ }^{(8)}$.

A população de estudo foi composta por estomizados provisórios cadastrados no referido Serviço de Atenção ao Estomizado e que atenderam aos seguintes critérios de inclusão e exclusão: idade igual ou superior a 18 anos; não possuir alterações cognitivas e mentais que impossibilitasse a participação, não ser acamado e que consentiram com a participação voluntária por meio da assinatura do Termo de Consentimento Livre e Esclarecido (TCLE).

Para proceder a coleta de dados, inicialmente foram levantados todos os prontuários dos estomizados cadastrados no referido Serviço e, a partir destes, selecionados aqueles com estoma provisório ou temporário. Assim, das 470 pessoas com estoma cadastrados no serviço, 117 (24,89\%) possuíam estoma provisório e enquadraram-se nos critérios de inclusão, correspondendo à amostra final do estudo.

A coleta de dados ocorreu por meio de fontes de dados primários (entrevista estruturada) e secundários, no período de setembro a novembro de 2015. Para a realização das entrevistas, as pessoas com estoma provisório eram abordadas na medida em que compareciam ao serviço, aplicando-se instrumento de coleta de dados estruturado, elaborado pelos próprios pesquisadores com base em revisão da literatura,, contendo variáveis sociodemográficas (sexo, idade, estado civil, escolaridade, religião, ocupação atual, afastamento da ocupação pós cirurgia, comorbidades como: doenças cardiovasculares e crônicas como hipertensão arterial sistêmica (HAS), diabetes mellitus (DM), dispidemias e suas consequências, infarto agudo do miocárdio (IAM) e acidente vascular cerebral (AVC) e renda familiar) e clínicas (data da realização do estoma, patologia, tempo com estoma, tipo de estoma, motivo de não reconstrução e tipo e quantidade de equipamento que faz uso). Previamente à coleta de dados, tal instrumento foi pré-testado em estudo piloto, com 10 pacientes, com o objetivo de verificar a adequabilidade do mesmo quanto à especificidade e clareza das variáveis abordadas, evitando possível viés de não entendimento ou influência do entrevistador. Ao final do pré-teste, o instrumento não foi modificado e as entrevistas foram incluídas na amostragem final.

Embora não haja consenso na literatura sobre o tempo ideal para reconstrução de estoma, considera-se clinicamente a possibilidade para reconstrução de trânsito intestinal a partir de quatro a sei meses pós-construção. Neste estudo, considerou-se "demora para a reconstrução" a permanência por mais de seis meses com o estoma.

Os dados foram inicialmente armazenados em planilha do aplicativo Excel, utilizando-se a técnica de dupla digitação. Após verificação de erros de transcrição, foram exportados para o Software Minitab 17 (Minitab Inc.), por meio do qual foram realizadas as análises do estudo.

Os dados foram analisados por meio de estatística descritiva e/ou analítica. Para verificar a associação, utilizamos o teste qui-quadrado entre as variáveis, motivos da demora da reconstrução do trânsito intestinal em relação à patologia, tipo de cirurgia, caráter da cirurgia e tipo de estoma.

Para verificar a associação entre a idade e tempo com o estoma com o tipo de patologia que levou à confecção do estoma, tipo de cirurgia realizada, tipo de estoma, caráter da cirurgia, motivos da demora para a reconstrução e retorno ao trabalho aplicou-se a Análise de Variância (ANOVA) e teste de Mann-Whitney, com teste de comparação múltipla de Tukey ou Games-Howell post-hoc. Utilizou-se nível de significância de 5\% na aplicação de todos os testes 
Este estudo foi aprovado pelo Comitê de Ética em Pesquisa (CEP) envolvendo seres humanos da Faculdade de Medicina de São José do Rio Preto (FAMERP), sob parecer $n$ o 1.228.105, seguindo as recomendações da Resolução $n^{\circ}$ 466/2012 do Conselho Nacional de Saúde.

\section{RESULTADOS}

As características sociodemográficas e clínicas dos participantes do estudo estão descritas na Tabela 1.

Tabela 1: Caracterização das pessoas estomizadas. São José do Rio Preto, SP, Brasil, 2015.

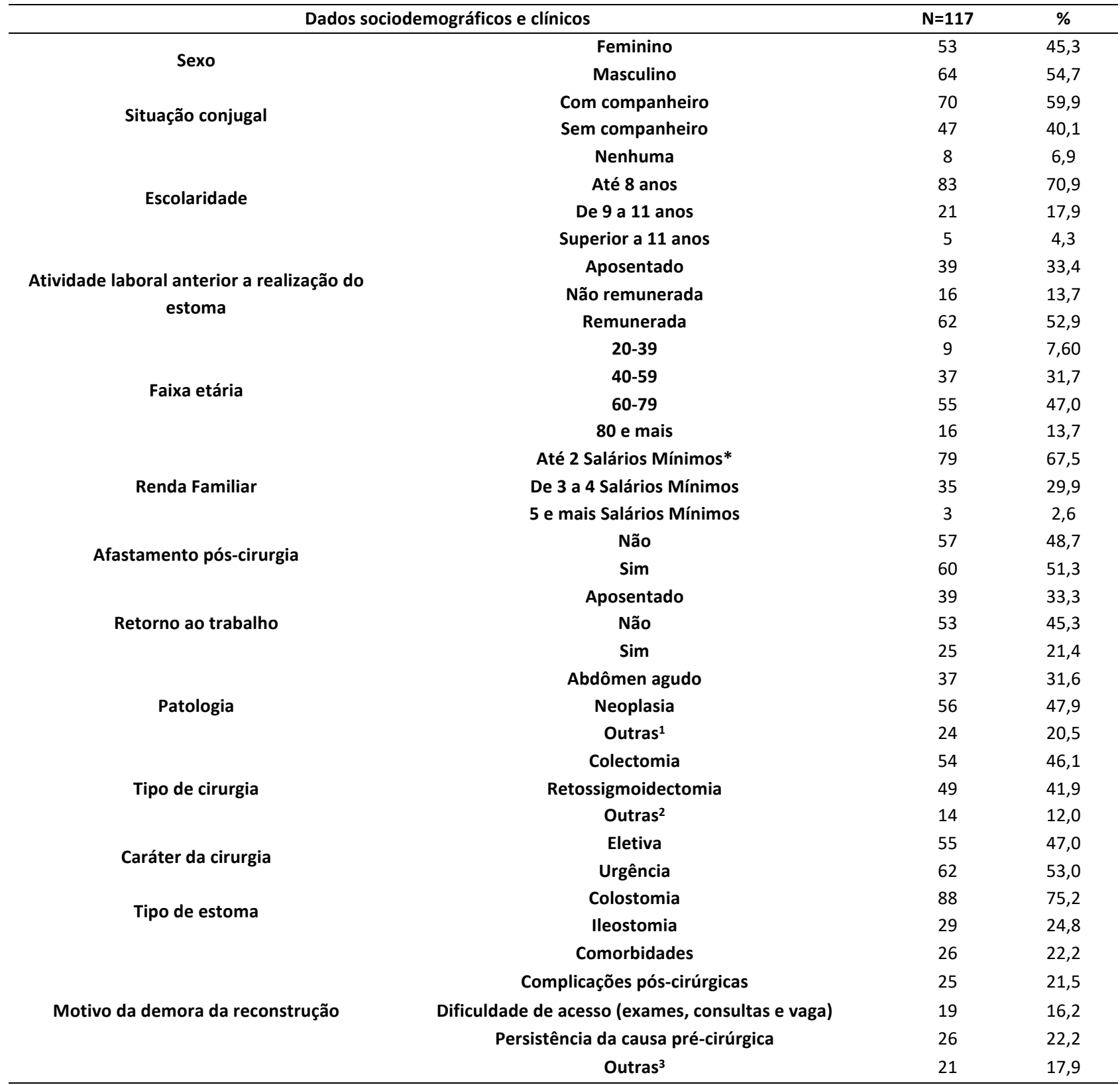

Legenda: Valor do salário mínimo: R\$788,00 conforme decreto 8381/2014 publicado no diário oficial da união em 30/12/2014; ${ }^{1}$ Agrupados: fístula, síndrome de Fournier, causas externas, doenças inflamatórias intestinais e doença de chagas sem perfuração ou obstrução do colón; ${ }^{2}$ Agrupados: laparotomia exploratória, enterectomia e ileotíflectomia; ${ }^{3}$ Agrupados: acostumou com a estomia, medo da cirurgia, contraindicação do médico.

Verificou-se que a idade interfere de forma significativa, tanto na patologia como no motivo da demora na reconstrução do trânsito intestinal $(p<0,05)$. Na análise da idade em relação à patologia, verificou-se que as pessoas estomizadas por abdômen agudo e neoplasia apresentaram idade significativamente superior em relação às pessoas que apresentaram outros tipos de patologias (Tabela 2). 
As pessoas estomizadas com comorbidades apresentaram idade significativamente superior em relação as que apresentaram os demais motivos de não reconstrução.

Tabela 2: Resultados da comparação da idade com as demais variáveis concernentes as pessoas estomizadas avaliadas no estudo. São José do Rio Preto, SP, Brasil, 2015.

\begin{tabular}{|c|c|c|c|c|c|}
\hline \multirow{2}{*}{\multicolumn{2}{|c|}{ Variáveis }} & \multicolumn{3}{|c|}{ Idade } & \multirow{2}{*}{ Valor $\mathrm{P}$} \\
\hline & & \multirow{2}{*}{\begin{tabular}{c|}
$\mathbf{N}$ \\
37
\end{tabular}} & \multirow{2}{*}{$\begin{array}{l}\text { Média } \pm D P \\
68,3 \pm 13,2 \mathrm{a}\end{array}$} & \multirow{2}{*}{$\begin{array}{c}\text { Mediana } \\
69,0\end{array}$} & \\
\hline & Abdômen agudo & & & & \\
\hline Patologia & Neoplasia & 56 & $64,5 \pm 11,6$ a & 66,5 & $<0,001$ \\
\hline \multirow{6}{*}{$\begin{array}{l}\text { Motivo da demora da } \\
\text { reconstrução }\end{array}$} & Outras & 24 & $51,2 \pm 16,3 b$ & 52,0 & \\
\hline & Comorbidades & 26 & $72,6 \pm 12,5 \mathrm{a}$ & 77,0 & \\
\hline & Complicações pós-cirúrgicas & 25 & $62,2 \pm 13,9 b$ & 62,0 & \multirow{4}{*}{$<0,001$} \\
\hline & $\begin{array}{c}\text { Dificuldade de acesso } \\
\text { (exames, consultas e vaga) }\end{array}$ & 19 & $56,1 \pm 14,5 b$ & 56,0 & \\
\hline & $\begin{array}{c}\text { Persistência da causa pré- } \\
\text { cirúrgica }\end{array}$ & 26 & $56,9 \pm 13,9 a b$ & 60,0 & \\
\hline & Outras & 21 & $65,7 \pm 11,3 b$ & 68,0 & \\
\hline
\end{tabular}

Legenda: ${ }^{1}$ Valor $p$ referente ao teste de Análise de Variância (ANOVA) a $\mathrm{P}<0,05$; Letras diferentes na mesma coluna indicam diferenças significativas pelo teste de comparação múltipla de Tukey a $\mathrm{P}<0,05$.

Na comparação entre o tempo com o estoma e a patologia $(p=0,099)$, tipo de cirurgia $(p=0,342)$, tipo de estoma $(p=0,709)$ e caráter da cirurgia $(p=0,590)$, não encontrou-se diferenças estatisticamente significante, conforme apresentado na Tabela 3.

Tabela 3: Resultados da comparação do tempo com estoma com as demais variáveis das pessoas avaliadas no estudo. São José do Rio Preto, SP, Brasil, 2015.

\begin{tabular}{|c|c|c|c|c|c|}
\hline & \multirow{2}{*}{ Variáveis } & \multicolumn{3}{|c|}{ Tempo com estoma (anos) } & \multirow{2}{*}{ Valor $\mathbf{P}^{1}$} \\
\hline & & $\mathbf{N}$ & Média $\pm D P^{2}$ & Mediana & \\
\hline \multirow{5}{*}{ Patologia } & Abdômen agudo & 37 & $6,3 \pm 5,0$ & 5,2 & \multirow{5}{*}{$0,099^{1}$} \\
\hline & Neoplasia & 56 & $3,9 \pm 4,4$ & 2,2 & \\
\hline & Outras & 24 & $5,9 \pm 5,9$ & 3,6 & \\
\hline & Comorbidades & 26 & $7,5 \pm 4,7$ a & 6,1 & \\
\hline & Complicações pós-cirúrgicas & 25 & $4,9 \pm 3,6$ a & 4,1 & \\
\hline \multirow[t]{4}{*}{$\begin{array}{l}\text { Motivo da demora } \\
\text { da reconstrução }\end{array}$} & Dificuldade de acesso (exames, consultas e vaga) & 19 & $1,3 \pm 1,3 b$ & 1,1 & \multirow[t]{4}{*}{$<0,001$} \\
\hline & Persistência da causa pré-cirúrgica & 26 & $2,2 \pm 2,5 b$ & 8,9 & \\
\hline & Outras & 21 & $9,4 \pm 6,5$ a & 1,1 & \\
\hline & Colectomia & 54 & $5,8 \pm 5,3$ & 3,6 & \\
\hline \multirow[t]{2}{*}{ Tipo de cirurgia } & Retossigmoidectomia & 49 & $4,9 \pm 5,2$ & 2,4 & \multirow[t]{2}{*}{$0,342^{1}$} \\
\hline & Outras & 14 & $3,2 \pm 2,8$ & 1,7 & \\
\hline \multirow{2}{*}{ Tipo de estoma } & Colostomia & 88 & $5,1 \pm 5,2$ & 2,8 & \multirow{2}{*}{$0,709^{3}$} \\
\hline & Ileostomia & 29 & $4,9 \pm 4,4$ & 2,4 & \\
\hline \multirow{2}{*}{ Caráter da cirurgia } & Eletiva & 55 & $4,8 \pm 4,9$ & 2,5 & \multirow{2}{*}{$0,590^{3}$} \\
\hline & Urgência & 62 & $5,4 \pm 5,1$ & 2,8 & \\
\hline \multirow{3}{*}{ Retorno ao trabalho } & Inativo & 39 & $6,1 \pm 4,5$ a & 5,5 & \multirow{3}{*}{$0,041^{1}$} \\
\hline & Não & 53 & $3,8 \pm 4,3 b$ & 1,3 & \\
\hline & Sim & 25 & $6,3 \pm 6,6$ a & 2,2 & \\
\hline
\end{tabular}

Legenda: ${ }^{1}$ Valor $\mathrm{P}$ referente ao teste de Análise de Variância a $\mathrm{P}<0,05 ;{ }^{2}$ Letras diferentes na mesma coluna indicam diferenças significativas pelo teste de comparação múltipla de Games-Howell, assumindo variâncias diferentes, a $\mathrm{P}<0,05 ;{ }^{3}$ Valor $\mathrm{P}$ referente ao teste de Mann-Whitney a $\mathrm{P}<0,05$.

Em contrapartida, o tempo com estoma apresentou diferenças estatisticamente significativas quando comparado ao motivo da demora na reconstrução do trânsito $(p<0,001)$ e ao retorno ao trabalho $(p=0,041)$.

As comorbidades, as complicações pós-cirúrgicas e a persistência da causa pré-cirúrgica foram os motivos da demora da reconstrução do trânsito daqueles que apresentaram maior tempo com estoma. As pessoas que relataram 
dificuldade de acesso como motivo para a demora na reconstrução do trânsito intestinal apresentaram menor tempo com estoma (Tabela 3).

O tempo com estoma das pessoas inativas e das que retornaram as atividades laborais foi significativamente superior ao daquelas que não retornaram ao trabalho (Tabela 3).

A Tabela 4 apresenta os percentuais de associação entre tais variáveis mencionadas.

Tabela 4: Associação dos motivos da demora de reconstrução do trânsito intestinal em relação à patologia, tipo de cirurgia, caráter da cirurgia e tipo de estoma. São José do Rio Preto/São Paulo, Brasil, 2015.

\begin{tabular}{|c|c|c|c|c|c|c|c|c|c|c|c|}
\hline \multirow{2}{*}{\multicolumn{2}{|c|}{ Variáveis qualitativas }} & \multicolumn{10}{|c|}{ Motivo da demora para reconstrução } \\
\hline & & \multicolumn{2}{|c|}{ Comorbidades } & \multicolumn{2}{|c|}{$\begin{array}{l}\text { Complicações pós- } \\
\text { cirúrgicas }\end{array}$} & \multicolumn{2}{|c|}{$\begin{array}{l}\text { Dificuldade de } \\
\text { acesso }\end{array}$} & \multicolumn{2}{|c|}{$\begin{array}{c}\text { Persistência da } \\
\text { causa pré-cirúrgica }\end{array}$} & \multicolumn{2}{|c|}{ Outras } \\
\hline & & N26 & $\%$ & N25 & $\%$ & N19 & $\%$ & N26 & $\%$ & N21 & $\%$ \\
\hline \multirow{5}{*}{ Patologia } & Abdômen agudo & 14 & 37,8 & 4 & 10,8 & 8 & 21,6 & 2 & 5,4 & 9 & 24,3 \\
\hline & Neoplasia & 10 & 17,9 & 15 & 26,8 & 8 & 14,3 & 17 & 30,4 & 6 & 10,7 \\
\hline & Outras & 2 & 8,3 & 6 & 25,0 & 3 & 12,5 & 7 & 29,2 & 6 & 25,0 \\
\hline & Valor $\mathbf{P}^{1}$ & \multicolumn{10}{|c|}{0,004} \\
\hline & Colectomia & 10 & 18,5 & 15 & 27,8 & 4 & 7,4 & 11 & 20,4 & 14 & 25,9 \\
\hline \multirow{3}{*}{$\begin{array}{l}\text { Tipo de } \\
\text { cirurgia }\end{array}$} & Retossigmoidectomia & 12 & 24,5 & 8 & 16,3 & 10 & 20,4 & 13 & 26,5 & 6 & 12,2 \\
\hline & Outras & 4 & 28,6 & 2 & 14,3 & 5 & 35,7 & 2 & 14,3 & 1 & 7,1 \\
\hline & Valor $\mathbf{P}^{1}$ & \multicolumn{10}{|c|}{0,092} \\
\hline \multirow{3}{*}{$\begin{array}{l}\text { Tipo de } \\
\text { estoma }\end{array}$} & Colostomia & 22 & 25,0 & 18 & 20,5 & 17 & 19,3 & 18 & 20,5 & 13 & 14,8 \\
\hline & Ileostomia & 4 & 13,8 & 7 & 24,1 & 2 & 6,9 & 8 & 27,6 & 8 & 27,6 \\
\hline & Valor $\mathbf{P}^{1}$ & \multicolumn{10}{|c|}{0,180} \\
\hline \multirow{3}{*}{$\begin{array}{l}\text { Caráter da } \\
\text { cirurgia }\end{array}$} & Eletiva & 11 & 20,0 & 11 & 20,0 & 6 & 10,9 & 16 & 29,1 & 11 & 20,0 \\
\hline & Urgência & 15 & 24,2 & 14 & 22,6 & 13 & 20,9 & 10 & 16,1 & 10 & 16,13 \\
\hline & Valor $\mathbf{P}^{1}$ & \multicolumn{10}{|c|}{0,326} \\
\hline
\end{tabular}

Legenda: ${ }^{1}$ Valor $\mathrm{P}$ referente ao teste qui-quadrado a $\mathrm{P}<0,05$.

Os resultados indicam a presença de uma associação significante entre os motivos da demora na reconstrução do trânsito intestinal e o tipo de patologia $(p=0,004)$. As pessoas que tiveram abdômen agudo apresentaram, em sua maioria, comorbidades como principal motivo para a demora da reconstrução; aquelas com neoplasia e outras patologias apresentaram como principal motivo da demora na reconstrução do trânsito a persistência da causa précirúrgica.

Houveram casos de pessoas com outros motivos de demora na reconstrução do trânsito, no entanto, tais percentuais não foram relevantes. As demais associações não foram estatisticamente significantes.

A análise sobre a influência da patologia e do tipo de estoma para o retorno ao trabalho indicou ausência de associação estatisticamente significante tanto para a patologia $(p=0,633)$ como para o tipo de estoma $(p=0396)$.

\section{DISCUSSÃO}

A maioria da amostra ser do sexo masculino pode estar relacionado à cultura dessa população, de não realizar medidas preventivas, acompanhamento de rotina e não valorizar sintomas iniciais, retardando a procura de auxílio, por se julgarem invulneráveis. As dificuldades de acesso aos serviços de saúde gerados tanto pela carga de trabalho, como também pelo horário de funcionamento de unidades básicas de saúde, que frequentemente é o mesmo do trabalhador, também contribuem para essa realidade. Esse retardo na busca por auxílio leva-os inúmeras vezes a entrar no sistema de saúde pela urgência devido à exacerbação de um sintoma que não foi valorizado anteriormente, determinando em muitos casos a necessidade de procedimento cirúrgico também de urgência ${ }^{(9)}$. Como foi possível evidenciar, a maioria dos procedimentos cirúrgicos foi realizado em caráter de urgência, tendo como causa a neoplasia, que quando 
diagnosticada a tempo pode ser realizada intervenção em caráter eletivo. Reforçando a necessidade de aprimorar os processos de prevenção, orientação e detecção precoce das neoplasias ${ }^{(10-11)}$.

A idade é fator que exerce influência significativa na patologia que determinou a construção do estoma. A mudança do cenário epidemiológico, com aumento na expectativa de vida, aliados a hábitos de vida não saudáveis contribuem para o aumento das doenças crônicas no Brasil, dentre elas as neoplasias ${ }^{(10)}$.

Segundo o Instituto Nacional de Câncer (INCA), o risco de desenvolver neoplasia aumenta com a idade, a maioria dos casos ocorre em adultos de meia idade ou idosos. A mesma instituição, estimou para o biênio 2016-2017 para a região sudeste, que a neoplasia do colón seria a segunda mais prevalente em ambos os sexos, ficando atrás apenas do câncer de próstata e mama, não incluindo o câncer de pele $\mathrm{e}^{(11)}$.

A idade também exerce influência para a não reconstrução de trânsito intestinal. As pessoas estomizadas que não reconstruíram o trânsito devido à comorbidades apresentaram idade superior quando comparadas com as demais causas. O envelhecimento do indivíduo é acompanhado pelo desenvolvimento de condições crônicas de saúde, essas morbidades podem aumentar a morbimortalidade na reconstrução do trânsito, culminando na não realização do procedimento em virtude dos riscos ${ }^{(10)}$.

Aspectos sociodemográficos e clínicos semelhantes foram encontrados em outros estudos realizados em diferentes regiões do Brasil( ${ }^{(12-17)}$, assim como na Europa ${ }^{(18)}$ e Ásia ${ }^{(19)}$.

A maior parte da população exercia alguma atividade laboral remunerada antes do procedimento cirúrgico que levou à construção do estoma. Analisando essa população pode-se vislumbrar que as pessoas estomizadas que retornaram ao trabalho possuíam maior tempo com estoma do que as que não retornaram, o que pode estar relacionado com dificuldades para o reingresso no mercado de trabalho. O retorno ao trabalho é uma importante ferramenta no processo de reabilitação, contribuindo no resgate da autoestima e auxiliando tanto na própria subsistência como na de sua família ${ }^{(15)}$.

A legislação favorece o retorno da pessoa com estoma ao trabalho incluindo-a como deficiente físico ${ }^{(20)}$. Pelo exposto se fazem necessários o planejamento e execução de procedimentos mais efetivos, por parte dos setores, instituições e pessoas envolvidas no processo de reabilitação, para que o retorno ao trabalho não seja tão moroso. As condições de trabalho devem ser adequadas à pessoa com estoma, seja no exercício da função realizada anterior a cirurgia, seja em função adaptada quando necessário, devido à natureza do trabalho exercido(15).

O tempo de permanência com estoma variou bastante, as comorbidades, complicações pós-cirúrgicas, e a persistência da causa pré-cirúrgica foram relatadas como motivo de demora para reconstrução pelas pessoas que possuíam estoma a mais tempo. A permanência do estoma provisório foi superior aos dados encontrados na literatura ${ }^{(2,3,19,21-22)}$.

Apesar de não existir consenso na literatura a respeito do tempo ideal para a reconstrução, alguns estudos trazem a possibilidade de reconstrução precoce nos casos de ileostomias de proteção(21). O uso de ileostomia para proteção de anastomose e seus benefícios para prevenir complicações no pós cirúrgico é bem definido entre os cirurgiões e no meio científico ${ }^{(22)}$. Neste estudo encontrou-se a prevalência de colostomias sobre as ileostomias. Este fato pode estar relacionado à técnica cirúrgica empregada, visto que houve prevalência da colectomia com estoma de uma boca, seguido da retossigmoidectomia do tipo Hartman; em ambas as técnicas foram realizadas construção de uma colostomia terminal.

Embora nos resultados, o tipo de cirurgia e de estoma não terem influenciado significativamente no aumento do tempo com estoma, a literatura destaca que, a reconstrução de trânsito em cirurgia do tipo Hartman possui 
consideráveis taxas de morbimortalidade, acarretando menor índice de reconstrução após esse procedimento. Portanto, deve-se analisar caso a caso a real necessidade de realização dessa técnica, restringindo sua indicação quando possível(5,19).

A patologia que determinou a construção do estoma foi significativa como causa de não reconstrução de trânsito intestinal. As pessoas com abdômen agudo apresentaram idade superior, e relataram comorbidades como principal motivo de demora para a reconstrução, dentre elas as principais foram complicações cardiovasculares, diabetes e hipertensão arterial. Estudo Europeu evidenciou a presença importante de doenças crônicas cardiovasculares ${ }^{(18)}$. Situação similar também foi encontrada na Ásia(19).

As pessoas com neoplasia e outras patologias apresentaram como motivo de não reconstrução a persistência da causa pré-cirúrgica. O tratamento das neoplasias compreende em grande parte dos casos o uso de quimioterapia ou radioterapia, além do procedimento cirúrgico. A recidiva da neoplasia ou persistência da mesma é apontada como fator de risco para a não reconstrução de trânsito em caso de estoma provisório ${ }^{(23)}$. Na categoria outras patologias foram agrupadas diversas patologias, como as doenças inflamatórias intestinais, doença de chagas e fístulas, que podem persistir mesmo com o tratamento adequado.

Embora a dificuldade no acesso a exames, consultas e para a própria cirurgia não tenha apresentado relevância estatística no aumento do tempo para reconstrução de trânsito, entende-se que esse achado pode estar refletindo as dificuldades geradas pela sobrecarga do sistema de saúde ${ }^{(24)}$. Como consequência ocorre o prolongamento do tempo como pessoa estomizada, impactando na qualidade de vida e aumentando os custos para o sistema de saúde. 0 aumento dos custos ocorre tanto pelo gasto com novos exames e avaliações médicas pré-cirúrgicas, como também pela aquisição de dispositivos, adjuvantes, manutenção de equipes qualificadas na atenção da pessoa com estoma, custos com afastamento previdenciário dentre outros que sobrecarregam e oneram ainda mais os sistemas governamentais.

Entre as principais limitações deste estudo pode-se citar o fato da não avaliação dos prontuários das instituições onde realizaram os estomas e avaliaram a reconstrução de trânsito, nos quais teriam dados importantes das reais causas de não reconstrução do trânsito intestinal. A inviabilização desse processo se deu devido ao tempo para execução, distância física entre instituições e a quantidade das mesmas.

Uma análise de prontuário desde a construção do estoma até as consultas de avaliação para reconstrução, em conjunto com entrevistas dos profissionais das equipes referência para reconstrução poderia trazer mais informações e elucidar questões que não foram esclarecidas neste primeiro momento.

\section{CONCLUSÃO}

Os principais motivos para a demora de reconstrução intestinal relatados foram a presença de comorbidades, persistência da causa pré cirúrgica, complicações pós cirúrgicas e dificuldade de acesso a exames, consultas e vaga de cirurgia. A idade é fator que influência de forma significativa tanto na patologia como no motivo de demora para reconstrução. A presença de comorbidades em decorrência do envelhecimento e a persistência da causa pré cirúrgica, principalmente no caso das neoplasias foram estatisticamente relevantes para a não reconstrução do trânsito intestinal. Esses fatores devem ser avaliados e sua interferência levada em consideração preferencialmente antes da construção do estoma, informando inclusive a pessoa e familiares.

O retorno tardio ao mercado de trabalho e a manutenção de serviços e materiais para assistência e reabilitação da pessoa com estoma possuem um custo que poderia ser reduzido com ações de integração de serviços e setores, com 
articulação em rede para potencializar tanto o retorno ao trabalho em condições adequadas, como a reconstrução de trânsito intestinal.

Acredita-se que campanhas preventivas e educativas sobre os fatores de risco, prevenção, sinais e sintomas e detecção precoce para neoplasias do colón, similares as já realizadas para câncer de mama e próstata poderia reduzir o número absoluto de casos e de cirurgias de urgência.

Acreditamos que a estruturação de um protocolo de cuidado em rede, com envolvimento e integração de todos os serviços pode contribuir na identificação de obstáculos, e na busca de soluções eficientes, melhorando a qualidade de vida das pessoas com estoma, qualificando o processo de reabilitação dos mesmos e reduzindo custos.

O enfermeiro estomaterapeuta, como integrante da equipe multidisciplinar contribui de forma singular no processo de reabilitação da pessoa com estoma, identificando situações para atuação da equipe, direcionando a assistência de forma humanizada. Esse profissional pode contribuir muito para a melhoria do cenário descrito, na estruturação, implantação e avaliação do protocolo que sugerimos.

\section{REFERÊNCIAS}

1. Rocha JJR. Estomas intestinais (ileostomias e colostomias) e anastomoses intestinais. Medicina (Ribeirao Preto. Online) [Internet]. 2011 [acesso em: 12 dez. 2018];44(1):51-6. Disponível em: https://doi.org/10.11606/issn.2176-7262.v44i1p51-56. 2. Silva JB, Costa DR, Menezes FJC, Tavares JM, Marques AG, Escalante RD. Perfil epidemiológico e morbimortalidade dos pacientes submetidos à reconstrução de trânsito intestinal: experiência de um centro secundário do Nordeste Brasileiro. Arq Bras Cir Dig [Internet]. 2010 [acesso em: 12 dez. 2018];23(3):150-3. Disponível em: https://doi.org/10.1590/S0102-67202010000300004. 3. Godat L, Kobayashi L, Chang DC, Coimbra R. Do Trauma Stomas Ever Get Reversed? J Am Coll Surg [Internet]. 2014 [acesso em: 12 dez. 2018];219(1):70-7.e1. Disponível em: https://doi.org/10.1016/j.jamcollsurg.2014.02.024.

4. Zafar SN, Changoor NR, Williams K, Acosta RD, Greene WR, Fullum TM, et al. Race and socioeconomic disparities in national stoma reversal rates. Am J Surg [Internet]. 2016 [acesso em: 12 dez. 2018];211(4):710-5. Disponível em:

https://doi.org/10.1016/j.amjsurg.2015.11.020.

5. Silva SM, Melo CCL, Almeida SB, Queiroz HF, Soares AF. Complicações das operações de reconstrução do trânsito intestinal. Rev bras. colo-proctol. [Internet]. 2006 [acesso em: 12 dez. 2018];26(1):24-7. Disponível em: https://doi.org/10.1590/S0101$\underline{98802006000100002 .}$.

6. Salomé GM, Almeida SA, Silveira MM. Quality of life and self-esteem of patients with intestinal stoma. J Coloproctology [Internet]. 2014 [acesso em: 12 dez. 2018];34(4):231-9. Disponível em: https://doi.org/10.1016/i.jcol.2014.05.009.

7. Sales CA, Violin MR, Waidman MAP, Marcon SS, Silva MAP. Sentimentos de pessoas ostomizadas: compreensão existencial. Rev Esc Enferm USP [Internet]. 2010 [acesso em: 12 dez. 2018];44(1):221-7. Disponível em: https://doi.org/10.1590/S008062342010000100031.

8. Portaria no 400 da Secretaria de Atenção à Saúde, de 16 de novembro de 2009 (BR) [Internet]. Estabelece diretrizes nacionais para a atenção à saúde das pessoas ostomizadas no âmbito do Sistema Único de Saúde - SUS, a serem observadas em todas as unidades federadas, respeitadas as competências das três esferas de gestão. Diário Oficial da União. 18 nov. 2009 [acesso em: 12 dez. 2018]. Disponível em: http://bvsms.saude.gov.br/bvs/saudelegis/sas/2009/prt0400_16_11_2009.html.

9. Ministério da Saúde, Secretaria de Atenção à Saúde, Departamento de Ações Programáticas e Estratégicas. Política Nacional de Atenção Integral à Saúde do Homem: princípios e diretrizes [Internet]. Brasília: Ministério da Saúde, 2009 [acesso em: 12 dez. 2018] Disponível em: http://portalarquivos2.saude.gov.br/images/pdf/2014/maio/21/CNSH-DOC-PNAISH---Principios-e-Diretrizes.pdf.

10. Ministério da Saúde, Secretaria de Vigilância em Saúde, Departamento de Analise de Situação de Saúde. Plano de ações estratégicas para o enfrentamento das doenças crônicas não transmissíveis (DCNT) no Brasil 2011-2022 [Internet]. Brasília: Ministério da Saúde, 2011 [acesso em: 12 dez. 2018]. Disponível em:

http://bvsms.saude.gov.br/bvs/publicacoes/plano acoes enfrent dcnt 2011.pdf.

11. Instituto Nacional de Câncer José Alencar Gomes da Silva, Coordenação de Prevenção e Vigilância. Estimativa 2016: incidência de câncer no Brasil. Rio de Janeiro: INCA, 2015.

12. Melotti LF, Bueno IM, Silveira GV, Silva MEN, Fedosse E. Characterization of patients with ostomy treated at a public municipal and regional reference center. J Coloproctology (Rio Janeiro) [Internet]. 2013 [acesso em: 12 dez. 2018];33(2):70-4. Disponível em: https://doi.org/10.1590/S2237-93632013000200005.

13. Souza APMA, Santos IBC, Soares MJGO, Santana IO. Perfil clínico-epidemiológico de los pacientes atendidos y censados en el Centro Paraibano de Ostomizados-João Pessoa, Brasil [Internet]. Gerokomos [Internet]. 2010 [acesso em: 12 dez. 2018];21(4):18390. Disponível em: http://scielo.isciii.es/scielo.php?script=sci arttext\&pid=S1134-928X2010000400007\&lng=es. 
14. Barbosa MH, Dal Poggetto MT, Barichello E, Cunha DF, Silva R, Alves PIC, et al. Aspectos clínicos e epidemiológicos de estomizados intestinais de um município de Minas Gerais. Revista de Enfermagem e Atenção à Saúde [Internet]. 2014 [acesso em: 12 dez. 2018];3(1):64-73. Disponível em: http://seer.uftm.edu.br/revistaeletronica/index.php/enfer/article/view/931.

15. Mauricio VC, Souza NVDO, Lisboa MTL. Determinantes biopsicossociais do processo de inclusão laboral da pessoa estomizada. Rev Bras Enferm [Internet]. 2014 [acesso em: 12 dez. 2018];67(3):415-21. Disponível em: https://doi.org/10.5935/00347167.20140055 .

16. Lins Neto MAF, Fernandes DOA, Didoné EL. Epidemiological characterization of ostomized patients attended in referral Center from the city of Maceió, Alagoas, Brazil. J Coloproctology [Internet]. 2016 [acesso em: 12 dez. 2018];36(2):64-8. Disponível em: https://doi.org/10.1016/i.jcol.2014.08.016.

17. Menezes LCG, Guedes MVC, Oliveira RM, Oliveira SKP, Meneses LST, Castro ME. Prática de autocuidado de estomizados: contribuições da teoria de Orem. Rev Rene [Internet]. 2013 [acesso em: 12 dez. 2018];14(2):301-10. Disponível em: http://periodicos.ufc.br/rene/article/view/3379.

18. Marquis P, Marrel A, Jambon B. Quality of life in patients with stomas: the Montreux Study. Ostomy Wound Manage [Internet]. 2003 [acesso em: 12 dez. 2018];49(2):48-55. Disponível em: https://www.o-wm.com/content/quality-life-patients-with-stomasthe-montreux-study.

19. Tan WS, Lim JF, Tang CL, Eu KW. Reversal of Hartmann's procedure: experience in an Asian population. Singapore Med J [Internet]. 2012 [acesso em: 12 dez. 2018];53(1):46-51. Disponível em: http://smj.sma.org.sg/5301/5301a6.pdf.

20. Decreto no 5.296, da Presidência da República, de 2 de dezembro de 2004 [Internet]. Regulamenta as Leis nos 10.048, de 8 de novembro de 2000, que dá prioridade de atendimento às pessoas que especifica, e 10.098, de 19 de dezembro de 2000, que estabelece normas gerais e critérios básicos para a promoção da acessibilidade das pessoas portadoras de deficiência ou com mobilidade reduzida, e dá outras providências. Diário da Oficial da União. 03 dez. 2004 [acesso em: 12 dez. 2018]. Disponível em: http://www.planalto.gov.br/ccivil_03/_Ato2004-2006/2004/Decreto/D5296.htm.

21. Danielsen AK, Correa-Marinez A, Angenete E, Skullmann S, Haglind E, Rosenberg J. Early closure of temporary ileostomy--the EASY trial: protocol for a randomised controlled trial. BMJ Open [Internet]. 2011 [acesso em: 12 dez. 2018];1(1):e000162. Disponível em: https://doi.org/10.1136/bmjopen-2011-000162.

22. Wu S-W, Ma C-C, Yang Y. Role of protective stoma in low anterior resection for rectal cancer: A meta-analysis. World J Gastroenterol [Internet]. 2014 [acesso em: 12 dez. 2018];20(47):18031-7. Disponível em:

https://doi.org/10.3748/wjg.v20.i47.18031.

23. Lee CM, Huh JW, Park YA, Cho YB, Kim HC, Yun SH, et al. Risk factors of permanent stomas in patients with rectal cancer after low anterior resection with temporary stomas. Yonsei Med J [Internet]. 2015 [acesso em: 12 dez. 2018];56(2):447-53. Disponível em: https://doi.org/10.3349/ymj.2015.56.2.447.

24. Von Bahten LC, Nicoluzzi JEL, Silveira F, Nicollelli GM, Kumagai LY, Lima VZ. Morbimortalidade da reconstrução de transito intestinal colônica em hospital universitário - análise de 42 casos. Rev Bras Coloproctol [Internet]. 2006 [acesso em: 12 dez. 2018];26(2):123-7. Disponível em: https://doi.org/10.1590/S0101-98802006000200002. 